Case Reports

\title{
Use of an Extremely Biodiverse Probiotic and a Supplement Based on Microbial Chondroitin Sulfate is Associated with a Significant Decrease of Serum Free Kappa Light Chains as well as a Trend Toward Normalization of Kappa/Lambda Ratio and of Plasma Cell Bone Marrow Infiltration in a Case of Multiple Myeloma
}

\author{
${ }^{1}$ Nicola Antonucci, ${ }^{2}$ Stefania Pacini and ${ }^{2}$ Marco Ruggiero \\ ${ }^{1}$ Biomedical Centre for Autism Research and Treatment, Bari, Italy \\ ${ }_{2}^{2}$ Silver Spring Sagl, Arzo-Mendrisio, Switzerland
}

Article history

Received: 27-05-2019

Revised: $13-06-2019$

Accepted: 18-06-2019

Corresponding Author:

Marco Ruggiero

Silver Spring Sagl, Via

Raimondo Rossi 24, Arzo-

Mendrisio 6864, Switzerland

E-mail: info@bravo-europe.com

\begin{abstract}
We describe the case of a 71-year-old woman with multiple myeloma associated with severe chronic renal insufficiency who, since October 2018, assumed a novel, extremely biodiverse, probiotic and a supplement based on microbial chondroitin sulfate, vitamin $\mathrm{D}_{3}$ and ultrapure phosphatidylcholine; such a nutritional regimen was associated with a dramatic decrease of serum free Kappa light chains (from 2,190 to a minimum of $119.40 \mathrm{mg} / \mathrm{L}$ ) as well as a trend toward normalization of Kappa/Lambda ratio (from 187 to a minimum of 3.16) and of plasma cell bone marrow infiltration (from 20 to $5 \%$ ).
\end{abstract}

Keywords: Multiple Myeloma, Probiotic, Colostrum, Phages, Chondroitin Sulfate, Vitamin $\mathrm{D}_{3}$, Phosphatidylcholine, RAD52

\section{Introduction}

Multiple myeloma is a neoplasia of plasma cells present in roughly $3-4 \%$ of the population over the age of 50 years and characterized by a cytogenetically heterogeneous plasma cell population. Multiple myeloma is considered an incurable hematologic neoplasia often requiring multiple sequential therapeutic regimens that have to be constantly tailored to the changing patient's conditions (Rajkumar et al., 2016). The standard of care for patients over 65 years and/or patients with serious concurrent chronic diseases is represented by chemotherapy with prednisone and melphalan or, more recently, with proteasome inhibitors (Hungria et al., 2019). An obstacle to chemotherapy of multiple myeloma is represented by renal insufficiency, a condition that is common in these patients and can often limit the choice of therapy (Mikhael et al., 2018).

Here we describe the case of a 71-year-old woman with multiple myeloma associated with severe chronic renal insufficiency who, since October 2018, assumed a novel, extremely biodiverse, probiotic and a supplement based on microbial chondroitin sulfate, vitamin $\mathrm{D}_{3}$ and ultrapure phosphatidylcholine; such a nutritional regimen was associated with a dramatic decrease of serum free Kappa light chains as well as a trend toward normalization of Kappa/Lambda ratio and of plasma cell bone marrow infiltration.

\section{Case Presentation}

The patient, a woman born in 1948, had been diagnosed with monoclonal gammopathy in 2010; in 2012 she developed acute renal insufficiency treated with dialysis and plasmapheresis. This event was followed by deep venous thrombosis with occlusion of the iliacfemoralis and femoralis-popliteal axes and systemic hypertension. Chronic renal insufficiency with frequent episodes of acute relapses resulted in extensive glomerular-tubular damage as evidenced by urinary protein electrophoresis; a renal biopsy documented advanced glomerulosclerosis with severe interstitial damage. In October 2018, the patient was admitted at the Oncohematological Department of the San Matteo hospital of Pavia, Italy; the document released by the 
hospital on October 29, 2018, reports that bone marrow biopsy of the posterior iliac crest confirmed a diagnosis of multiple myeloma with monotypical plasma cell bone marrow infiltration accounting for $20 \%$ of total cells. Study of serum free light chains showed significant alteration of the Kappa/Lambda ratio (free Kappa: 2,190 $\mathrm{mg} / \mathrm{L}$. Free Lambda: $11.7 \mathrm{mg} / \mathrm{L}$. Ratio Kappa/Lambda: 187) that was consistent with the severity of the oncohematological disease according to the updated criteria for the diagnosis of multiple myeloma (Rajkumar et al., 2014). Following hospitalization in October 2018, the patient opted for a complementary approach to her condition consisting in consumption of $50 \mathrm{~mL}$, twice a day, of an extremely biodiverse probiotic drink obtained by fermentation of bovine milk and colostrum; in addition, the Medical Doctor supervising the patient, in October 2018 begun administering $0.2 \mathrm{~mL}$ per week of a novel immune-modulating supplement based on microbial chondroitin sulfate, vitamin $\mathrm{D}_{3}$ and ultrapure phosphatidylcholine (Ruggiero, 2018). A laboratory exam performed three months later (January 10, 2019) demonstrated a 95\% decrease of serum free Kappa light chains that went from $2,190 \mathrm{mg} / \mathrm{L}$ (October 2018) to $119.40 \mathrm{mg} / \mathrm{L}$. Also the ratio Kappa/Lambda showed a trend toward normalization decreasing from 187 (October 2018) to 11.10 (January 2019). Consistent with the significant improvement of the oncohematological picture, also the renal function improved, albeit to a lesser extent. Creatinine decreased from $1.76 \mathrm{mg} / \mathrm{dL}$ (October 2018) to $1.61 \mathrm{mg} / \mathrm{dL}$ (January 2019); glomerular filtrate increased from 28 $\mathrm{mL} / \mathrm{min}$ (October 2018) to $34 \mathrm{~mL} / \mathrm{min}$ (January 2019). Two months later (March 1, 2019) the patient was admitted to the Hospital of Eboli, Italy, for a trauma to her left leg due to an accidental fall at home; the laboratory exams performed on that occasion demonstrated further improvement of the oncohematological condition with the ratio $\mathrm{Kappa} /$ Lambda decreased to 3.5 that is very close to the upper limit of normal values (2.93). A further laboratory exam performed about two months later (May 24, 2019) at the University Hospital San Giovanni di Dio e Ruggi d'Aragona, Salerno, Italy, confirmed the continuing descending trend of the Kappa/Lambda ratio that was 3.16. Consistent with these results, a bone marrow aspiration at the sternum performed on April 26, 2019 at the San Giuseppe Moscati Hospital, Avellino, Italy, evidenced plasma cell infiltration of $5 \%$ that is a very significant reduction in comparison to the results observed before implementing the nutritional approach described above; thus, plasma cell bone marrow infiltration was $20 \%$ in October 2018. The report from the San Giuseppe Moscati Hospital was consistent with a quasi-normal condition as it read: "Bone marrow aspiration. Slightly reduced bone density. Specimen very rich in cells and tiny lumps. Parenchymal erythroblasts and granulocytes are normal and in normal stage of maturation. Number of lymphocytes is normal. Very slight (in Italian, lievissimo) increase of plasma cells that are present in a percentage of about $5 \%$; some plasma cell is binucleate. Megakaryocytes are numerous and most of them in active terminal thrombopoiesis." (literal translation from Italian).

\section{Discussion}

A number of preclinical and clinical trials highlights the importance of the microbiota in cancer and there is general consensus that modulation of the microbiota plays a fundamental role in determining the response of cancer to therapies (Gopalakrishnan et al., 2018). The observation described in this case supports the hypothesis that a combination of a microbiota-based approach with immune-modulating strategies may prove effective in oncohematological diseases. It is worth noticing that the milk and colostrum fermented drink assumed by the patient here described represents a novel form of probiotic because of its extreme biodiversity; ingredients, microbial composition and nutritional characteristics of this drink are described in Table 1. A microbiome assay performed with a chip covered in DNA sequences that are specific to target organisms and is able to detect a total of approximately 12,000 species (Thissen et al., 2019), revealed the presence of hundreds of different microorganisms, thus showing an extremely high degree of biodiversity that included plasmids and phages with the latter being heralded as the tools for the future of immunotherapy (Rehman et al., 2019). It is known that phages are endowed with anticancer activity and it was demonstrated that they inhibit the growth of experimental melanoma and lung cancer and also inhibit the formation of lung metastases (Dabrowska et al., 2004). In addition, phages are responsible for activation of tumoricidal, classically activated, M1 macrophages and it was demonstrated that, following cancer treatment with phages, the M2-polarized tumor microenvironment switched toward an M1-polarized milieu (Eriksson et al., 2009). At the molecular level, an example highlighting the role of the phages is represented by the presence of Lactococcus lactis phage ul36 (Siphoviridae) in the probiotic drink assumed by the patient. As demonstrated since 2008, this microorganism encodes a single-strand annealing protein (Sak) that is homologous to the human recombination protein RAD52, a protein that plays crucial roles in DNA repair and genome stability (Ploquin et al., 2008). 
Table 1: Characteristics of the probiotic

Description of the product Ingredients, colostrum

Ingredients, microbial blends

Product based on bovine colostrum and live probiotics for home preparation of fermented milk

Freeze-dried, pasteurized bovine colostrum. 5 grams per liter of final product.

Blend of lyophilized kefir grains containing live cultures (including Streptococcus thermophilus and Lactobacillus delbrueckii, subspecie bulgaricus) and yeasts.

Blend of Bifidobacteria: Bifidobacterium infantis (25\%); Bifidobacterium bifidum (25\%); Bifidobacterium lactis (25\%); Bifidobacterium longum (25\%).

Blend of lyophilized micro-encapsulated live cultures; Bifidobacterium infantis (12.5\%); Bifidobacterium bifidum (12.5\%); Bifidobacterium lactis (12.5\%); Lactobacillus salivarius (12.5\%); Lactobacillus acidophilus (12.5\%); Lactobacillus casei, subspecie paracasei (12.5\%); Lactobacillus rhamnosus (12.5\%); Lactococcus lactis, subspecie cremoris $(12.5 \%)$. Coating agents for this blend (micro-encapsulation); mono- and diglycerides of fatty acids. Calories: 4 Kcal. Proteins: 0.28 grams. Fats: 0.28 grams. Carbohydrates: 0.22 grams. These values refer to the ingredients listed above in the measure required to prepare 1 liter of final product.

Nutritional information

The ingredients listed above are used by the final utilizer to ferment bovine milk at room temperature for $24 \mathrm{~h}$ and prepare a probiotic drink. The microbiome assay described in the text refers to the product of fermentation and not to the individual ingredients listed above. The microbiome assay was performed by an independent laboratory on the fermented probiotic drink that was lyophilized prior to the assay. We hypothesize that the extreme biodiversity mentioned in the text is due to the process of fermentation as well as to the complex microflora of the kefir grains.

From an evolutionary perspective, however, it is the human RAD52 protein that has evolved from the phage Sak protein, thus further emphasizing the role of phage-associated genetic information in maintaining DNA stability and, in a broader sense, human health.

It is tempting to speculate that the wide-range of effects on the immune system deriving from the extreme biodiversity of the probiotic drink assumed by the patient was synergistic with the effects of the supplement based on microbial chondroitin sulfate, vitamin $\mathrm{D}_{3}$ and ultrapure phosphatidylcholine as they are described in two recent papers (Ruggiero, 2018; Ruggiero and Pacini, 2018). Such a synergism may well be at work at the molecular level, for example by integrating different mechanisms responsible for DNA repair that counteract DNA instability, a common trait of cancer and other conditions. On one hand, the Sak/RAD52 pathway of the probiotic targets single-stranded DNAs preferentially over double-stranded DNAs and promotes the renaturation of long complementary single-stranded DNAs (Ploquin et al., 2008); on the other hand, the vitamin $\mathrm{D}_{3}$ of the supplement promotes expression of the DNA repair genes RAD50 and ATM (Ataxia Telangiectasia Mutated), that play a key role in mediating the signaling responses to double-strand DNA breaks (Ting et al., 2012). The convergence of these pathways maximizes the ability of cells to restore singleand double-strand DNA breaks thus efficiently counteracting a variety of DNA damages that are considered responsible for cancer onset and progression. It is also worth emphasizing the synergism between the phages in the probiotic and the phosphatidylcholine in the supplement in promoting and stabilizing a strong M1 phenotype in macrophage polarization (Qin et al., 2014).

Since this clinical case report is an open-label, noncontrolled, retrospective analysis, caution must be exercised when ascribing cause and effect to any treatment outcome. In addition, as of June 2019, the patient is assuming the following drugs in the context of chronic renal disease management; prednisone, $5 \mathrm{mg} / \mathrm{die}$; omeprazole, $20 \mathrm{mg} / \mathrm{die}$; folic acid, $5 \mathrm{mg} / \mathrm{die}$; febuxostat, $80 \mathrm{mg} / \mathrm{die}$; telmisartan, $20 \mathrm{mg}$ when needed; warfarin 5 mg administered according to the patient's INR. The patient did not report side effects associated with the nutritional approaches described above assumed in conjunction with her current therapy. Although prednisone may have contributed to the observed results as it is used in the therapy of multiple myeloma, it is worth noticing that the patient had been assuming this drug alongside others for a long time. In addition, the dose for the management of multiple myeloma is ten times higher than that assumed by the patient (Berenson et al., 2002). Despite these limitations, it appears that the association between the probiotic and the supplement and the improvements demonstrated by objective laboratory data was robust. It is well accepted that case reports are constituents of evidence-based medicine and informed practice (Juyal et al., 2013) and we are convinced that this novel and unusual clinical observation may lead to subsequent research advances in the field of complementary immunotherapy of cancer.

\section{Acknowledgements}

The Authors wish to thank the patient whose case is described here and her relatives for their priceless collaboration.

\section{Conflict of Interest}

Nicola Antonucci is the founder of the Biomedical Centre for Autism Research and Treatment, a private clinic. Marco Ruggiero is the inventor of a number of supplements and, together with Stefania Pacini, developed the probiotic (Bravo, Silver Spring Sagl, 
Switzerland) and the supplement (imuno ${ }^{\circledR}$, imuno Corporation, Vanuatu) used in this study. Both the probiotic and the supplement were regularly purchased and paid for by the patient. Neither Dr. Ruggiero, nor Dr. Pacini, had any prior knowledge of the strategies being implemented nor of the details of the patient whose clinical outcomes were analyzed and described in this study. Marco Ruggiero is member of the Editorial Board of The American Journal of Immunology and is waived from the Article Processing fee for this contribution; he receives no remuneration for his editorial work.

\section{Authors' Contribution}

Nicola Antonucci: Performed the therapeutic procedures described in this study and communicated the clinical and laboratory data of the patient.

Marco Ruggiero and Stefania Pacini: Wrote the first draft of this paper, provided critical input and assisted in revising and improving the paper.

\section{Ethics}

No information in this paper is presented by the authors as medical advice. Caregivers, researchers and interested parties should research all information given. Beginning any significant biomedical or other interventions that may impact physiology or making changes to an established regimen should be discussed with the patient's physician in advance. Standard of care for each pathology must be followed as well as rules and regulations established by Health Authorities of each Country.

\section{References}

Rajkumar, S.V., 2016. Myeloma today: Disease definitions and treatment advances. Am. J. Hematol., 1: 90-100. DOI: 10.1002/ajh.24236

Eriksson, F., P. Tsagozis and K. Lundberg, R. Parsa and S.M. Mangsbo et al., 2009. Tumor-specific bacteriophages induce tumor destruction through activation of tumor-associated macrophages. J. Immunol., 182: 3105-3111.

DOI: 10.4049/jimmunol.0800224

Ploquin, M., A. Bransi and E.R. Paquet, A.Z. Stasiak and A. Stasiak et al., 2008. Functional and structural basis for a bacteriophage homolog of human RAD52. Curr. Biol., 18: 1142-6.

DOI: $10.1016 /$ j.cub.2008.06.071

Ruggiero, M. and S.A. Pacini, 2018. Novel potential adjuvant for cancer vaccines. Madridge J. Vaccines, 2: 57-1. DOI: $10.18689 / \mathrm{mjv}-1000112$
Ting, H.J., S. Yasmin-Karim, S.J. Yan, J.W. Hsu and T.H. Lin et al., 2012. A positive feedback signaling loop between ATM and the vitamin D receptor is critical for cancer chemoprevention by vitamin D. Cancer Res., 72: 958-68.

DOI: 10.1158/0008-5472.CAN-11-0042

Qin, X., C. Qiu and L. Zhao, 2014. Lysophosphatidylcholine perpetuates macrophage polarization toward classically activated phenotype in inflammation. Cell Immunol., 289: 185-90.

DOI: 10.1016/j.cellimm.2014.04.01015

Juyal, D., S. Thaledi and V. Thawani, 2013. Thawani writing patient case reports for publication. Educ. Health, 26: 126-9. DOI: 10.4103/1357-6283.120707

Hungria, V.T.M., E.Q. Crusoé, R.I. Bittencourt, R.P. Magalhães and J.N. Sobrinho et al., 2019. New proteasome inhibitors in the treatment of multiple myeloma. Hematol. Transfus Cell Ther., 1: 76-83. DOI: $10.1016 /$ j.htct.2018.07.003

Mikhael, J., J. Manola, A.C. Dueck, S. Hayman and K. Oettel et al., 2018. Lenalidomide and dexamethasone in patients with relapsed multiple myeloma and impaired renal function: PrE1003, a PrECOG study. Blood Cancer J. DOI: 10.1038/s41408-018-0110-7

Rajkumar, S.V., M.A. Dimopoulos, A. Palumbo, J. Blade and G. Merlini et al., 2014. International myeloma working group updated criteria for the diagnosis of multiple myeloma. Lancet Oncol,. 15: e538-48. DOI: 10.1016/S1470-2045(14)70442-5

Ruggiero, M., 2018. Rationale for the design of a novel tool for immunotherapy based on an emulsion of glycosaminoglycan. Integr. Cancer Sci. Therap, 5: 1-5. DOI: 10.15761/ICST.1000285

Gopalakrishnan, V., B.A. Helmink, C.N. Spencer, A. Reuben and J.A. Wargo et al., 2018. The Influence of the gut microbiome on cancer, immunity and cancer immunotherapy. Cancer Cell, 33: 570-580. DOI: 10.1016/j.ccell.2018.03.015

Thissen, J.B., N.A. Be, K. McLoughlin, S. Gardner and P.G. Rack et al., 2019. Axiom microbiome array, the next generation microarray for high-throughput pathogen and microbiome analysis. PLoS ONE. DOI: 10.1371/journal.pone.0212045

Rehman, S., Z. Ali, M. Khan, N. Bostan and S. Naseem et al., 2019. The dawn of phage therapy. Rev. Med. Virol. DOI: 10.1002/rmv.2041

Dabrowska, K., A. Opolski, J. Wietrzyk, K. SwitalaJelen and J. Boratynski et al., 2004. Antitumor activity of bacteriophages in murine experimental cancer models caused possibly by inhibition of 3 integrin signaling pathway. Acta Virol., 48: 241-248. PMID: 15745047 
Berenson, J.R., J.J. Crowley, T.M. Grogan, J. Zangmeister and A.D. Briggs et al., 2002. Maintenance therapy with alternate-day prednisone improves survival in multiple myeloma patients. Blood, 99: 3163-8. DOI: 10.1182 /blood.V99.9.3163

\section{Accessory Material}

Clinical and laboratory records are archived at the Biomedical Centre for Autism Research and Treatment, Bari. Italy. Since this is a low-number case report that does not produce generalizable knowledge, nor an investigation of an FDA regulated product, Institutional Review Board (IRB) review is not required for this activity (John Hopkins Medicine. 102.3 Organization Policy on Single Case Reports and Case Series. https://www.hopkinsmedicine.org/institutional_review_b oard/guidelines_policies/organization_policies/102_3.ht ml. Web page accessed June 10, 2019). 